\title{
Using Bayesian methods for the parameter estimation of deformation monitoring networks
}

\author{
E. Tanir ${ }^{1,2}$, K. Felsenstein ${ }^{3}$, and M. Yalcinkaya ${ }^{2}$ \\ ${ }^{1}$ Vienna University of Technology, Institute of Geodesy and Geophysics, 1040 Vienna, Austria \\ ${ }^{2}$ Karadeniz Technical University, Department of Geodesy and Photogrammetry Engineering, 61080 Trabzon, Turkey \\ ${ }^{3}$ Vienna University of Technology, Institute of Statistics and Probability Theory, 1040 Vienna, Austria
}

Received: 24 October 2007 - Revised: 25 January 2008 - Accepted: 27 March 2008 - Published: 11 April 2008

\begin{abstract}
In order to investigate the deformations of an area or an object, geodetic observations are repeated at different time epochs and then these observations of each period are adjusted independently. From the coordinate differences between the epochs the input parameters of a deformation model are estimated. The decision about the deformation is given by appropriate models using the parameter estimation results from each observation period. So, we have to be sure that we use accurately taken observations (assessing the quality of observations) and that we also use an appropriate mathematical model for both adjustment of period measurements and for the deformation modelling (Caspary, 2000). All inaccuracies of the model, especially systematic and gross errors in the observations, as well as incorrectly evaluated a priori variances will contaminate the results and lead to apparent deformations. Therefore, it is of prime importance to employ all known methods which can contribute to the development of a realistic model. In Albertella et al. (2005), a new testing procedure from Bayesian point of view in deformation analysis was developed by taking into consideration prior information about the displacements in case estimated displacements are small w.r.t. (with respect to) measurement precision.

Within our study, we want to introduce additional parameter estimation from the Bayesian point of view for a deformation monitoring network which is constructed for landslide monitoring in Macka in the province of Trabzon in north eastern Turkey. We used LSQ parameter estimation results to set up prior information for this additional parameter estimation procedure. The Bayesian inference allows evaluating the probability of an event by available prior evidences and collected observations. Bayes theorem underlines that the observations modify through the likelihood function the
\end{abstract}

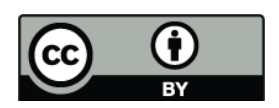

Correspondence to: E. Tanir (etanir@mars.hg.tuwien.ac.at) prior knowledge of the parameters, thus leading to the posterior density function of the parameters themselves.

\section{Introduction}

The technological progress during the last decades has also affected geosciences and the observational methods in all fields of geosciences have changed gradually. Therefore, today measurements for deformation monitoring are conducted commonly by satellite based techniques. Consequently, it becomes possible to make deformation monitoring studies in adequate accuracy in less time for larger areas. However, the increasing observational accuracy requires adequate mathematical and statistical models. Measurement errors occur no matter how measurements are taken by terrestrial and satellite techniques and have to be eliminated from the measurements. Determining measurement errors by effective measurement analysis is as important in deformation monitoring studies as determining the deformation model itself. In the last decade, attention has shifted towards Bayesian statistics, which has advantages in complex problems and better reflects the way scientists think about evidence. Recently, the Bayesian statistics has been used efficiently in the areas of engineering, social sciences and medicine (Koch 1990; Yalcinkaya and Tanir, 2003).

Bayes theorem leads to posterior distribution of unknown parameters given by the data. All inferential problems concerning the parameters can be solved by means of posterior distributions. Based on these distributions we will estimate the unknown parameters, establish confidence regions for them and test hypotheses concerning the parameters. In this study, it is aimed to apply Bayes-Updating (BU) and Gibbs-Sampling (GS) algorithms in a geodetic parameter estimation problem. In addition, the task is to inform the user which algorithm is more useful in the aspect of accuracy and

Published by Copernicus Publications on behalf of the European Geosciences Union. 
methodology. We investigated the sensitivity of these algorithms due to the use of different prior information.

To apply the Bayes-Updating we introduce the prior information for station coordinates. We started up with noninformative prior and used different epoch observations in different order to stepwisely update prior information. In Gibbs-Sampling algorithm we introduce different starting points w.r.t. point positioning errors. Usually, measurements at different points of a network are of different quality concerning their statistical variance. We test the quality of network points estimated coordinates by comparing the Bayesian estimates derived from a Bayes-Updating (conjugate updating) algorithm or a Gibbs-sampling procedure. The total variation (L1-distance) of the resulting estimates serves as measure of discrepancy for reasons of robustness. The information contained in a network point is evaluated by taking that point as starting point for Gibbs-sampling. The distances (L1-measure) between Bayesian updating and Gibbs-sampling indicate how much imprecision the point adds to the estimation procedure.

We consider the information contained in different data sets as well as the influence of one single network point. The differences of estimation taking data sets in exchanging order (concerning prior data set and actual data set) serves as method to evaluate the information contained in the data set.

\section{Bayesian parameter estimation methods}

\subsection{Bayes updating}

The basic instrument of Bayesian procedures consists of the updating algorithm via Bayes theorem

$\pi(\theta \mid D) \propto l(\theta, D) \pi(\theta)$

where $\pi(\theta)$ denotes the prior distribution and $\pi(\theta \mid D)$ the posterior distribution of the model parameter. $l(\theta, D)$ denotes the likelihood function of the data. For the network data we use a correlated normal vector $\boldsymbol{y}$ with the mean vector $\boldsymbol{\mu}$ and variance-covariance matrix $\Sigma$. In a conjugate prior approach the prior knowledge about parameters is assessed in the same form as the likelihood. In case of correlated normal observations the corresponding prior is a NormalWishart distribution (Felsenstein, 1996). The common prior of $\mu$ and the precision matrix $\mathbf{P}=\Sigma^{-1}$ is split into a normal part as a normal distribution with a mean of $m$ and standard deviation of $(b \mathbf{P})^{-1}$

$\boldsymbol{\mu} / \mathbf{P} \sim N\left(\boldsymbol{m},(b \mathbf{P})^{-1}\right)$

with $b>0$ and Wishart part

$\mathbf{P}=\Sigma^{-1} \sim \operatorname{Wis}(a, \Lambda)$

The weights $b, a>0$ play the same role as the number of observations in the likelihood. $\boldsymbol{\mu}$ is the prior guess for the mean and $\Lambda$ a prior guess for the covariance matrix. For the data of the geometric network it is important to specify the prior covariance according to the geometric structure $(\mathrm{Eu}-$ clidian distances on surfaces). Once the prior hyper parameters are specified the posterior can be achieved through the updating of the hyper parameters only. The posterior density is

$\pi(\boldsymbol{\mu}, \mathbf{P} \mid D)=\frac{f(D \mid \boldsymbol{\mu}, \mathbf{P}) \pi(\boldsymbol{\mu} \mid \mathbf{P}) \pi(\mathbf{P})}{m(D)}$

where $f$ denotes the multivariate Normal distribution with mean $\boldsymbol{\mu}$ and precision matrix $\mathbf{P}$. The marginal density is

$m(D)=\int f(D \mid \boldsymbol{\mu}, \mathbf{P}) \pi(\boldsymbol{\mu} \mid \mathbf{P}) \pi(\mathbf{P}) d \boldsymbol{\mu} d \mathbf{P}$

For more details see also Rowe 2002. The hyper parameters are computed out of data vectors as following by

$\boldsymbol{m}^{*}=\frac{b \boldsymbol{m}+n \overline{\boldsymbol{y}}}{b+n}$

and

$\Lambda^{*}=\Lambda+\frac{n b}{b+n}(m-\bar{y})(m-\bar{y})^{T}+R$

where $\mathbf{R}$ is the empirical covariance

$\mathbf{R}=\sum_{i} \boldsymbol{y}_{i} \boldsymbol{y}_{i}^{T}-n \overline{\boldsymbol{y}}^{T}$

The Eqs. (6), (7) and (8) are considered as updating algorithm for current epoch of a geodetic network with a priori data set of the same network from a different epoch with $\boldsymbol{m}^{*}$ posterior mean for current data epoch, $b$ number of observations of different epochs which prior information comes from, $\boldsymbol{m}$ observations' prior mean of different epochs which prior information comes from, $n$ number of observations of current epoch. $\Lambda$ variance-covariance matrix of observations of different epoch which prior information comes from, $\Lambda^{*}$ posterior variance-covariance matrix of observations of current epoch. The modelling through a conjugate family of priors is indeed attractive even in case of little informative prior information. Prior distributions carrying as little information as possible can be considered as limits of conjugate priors in the sense

$\Lambda \rightarrow 0$

and $b \rightarrow 0$. The conjugate property is not restricted to a single distribution. Mixtures of Normal-Wisharts are conjugate as well allowing more flexibility to the model. For weighting we choose several values of $b$ in our calculations to analyze the sensitivity of the estimates upon the prior distributions (Robert, 2001). Bayesian estimates of the different parameters of the network are calculated as posterior means leading to weighted mixtures of the means

$\boldsymbol{m}_{j}^{*}=\frac{b \boldsymbol{m}_{j}+n \overline{\boldsymbol{y}}}{b+n}$ 


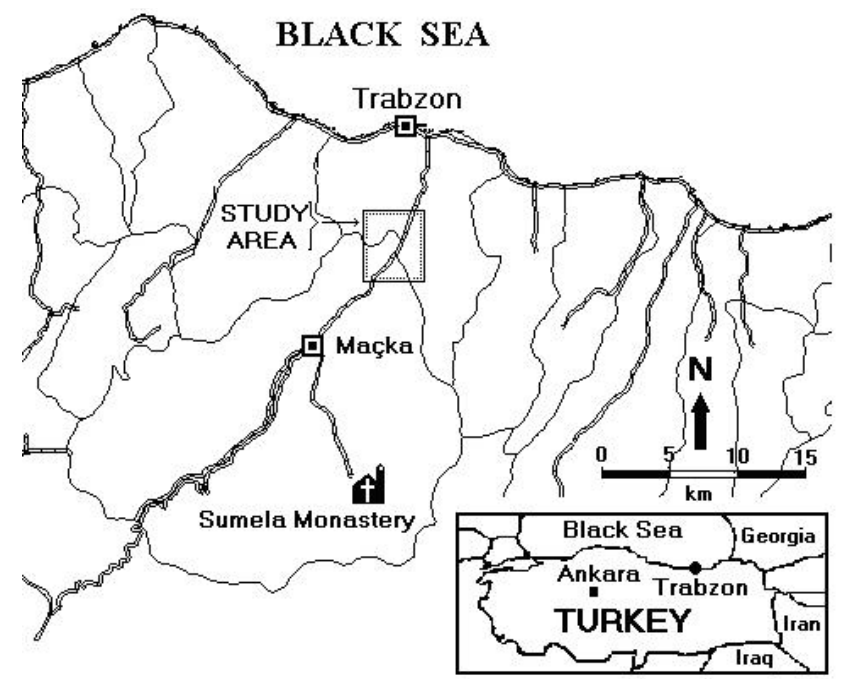

Fig. 1. Location map of study area (Yalcinkaya and Bayrak, 2003).

as the posterior expectations of $\boldsymbol{\mu}$. Here $\mathrm{j}$ indicates one Normal-Wishart distribution out of the mixture. $a_{j}$ and $b_{j}$ are altered according to $a^{*}=a+n$ and $b^{*}=b+n$. The Bayesian estimate of the covariance is

$E(\Sigma / D)=\frac{1}{a+n-k-1} \Lambda^{*}$

where $k$ is the dimension ( $k=14$, number of network points in this study). Since the specification of a completely informative prior turns out to be practically impossible for the covariance structure we choose an approach which can be compared to a modified empirical Bayes setup. First we take one data set as a learning sample equipped with non-informative prior. By this first step we achieve a reliable prior guess of $\Sigma$ used as $\Lambda$. Namely, we insert the empirical covariance matrix of the learning sample as prior covariance matrix. In alternating these starting sets and comparing the results we analyze the sensitivity of the model concerning the prior covariance structure. Note that Bayesian estimates coincide with standard least-squares estimates in case of non-informative priors.

\subsection{Gibbs-sampling}

Since all measurements in the network are highly correlated the distributions (posterior as well as prior) of the parameters become high dimensional. Therefore an algorithm is needed to handle the posterior distributions which incorporate the special covariance structure of the data. The generic Gibbs algorithm aims at reproducing the posterior density and associated estimates in an automatic manner. Gibbs sampling represents a specific application of general MCMC (Markov Chain Monte Carlo) processes (Robert and Casella, 2004).

The Gibbs sampling algorithm works as follows. The distribution of the $k$ dimensional stochastic variable

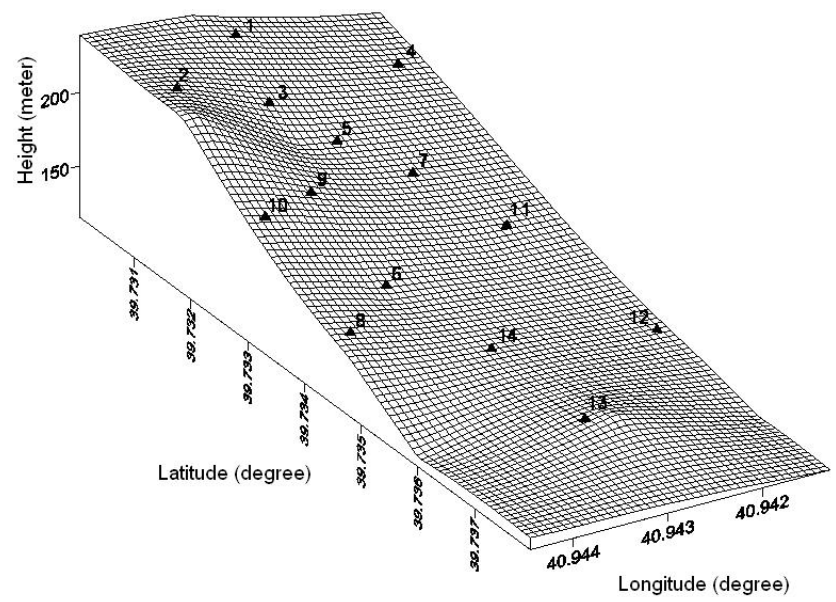

Fig. 2. 3D-view of the study area.

$\boldsymbol{y}=\left(y_{1}, \ldots, y_{k}\right)$ is simulated by the following transition from step $s$ to $s+1$. The basic idea of the algorithm is the simulation of the full conditional distributions where the components of the stochastic vector are replaced in turn. Let $y_{1}^{[0]}, \ldots, y_{k}^{[0]}$ denote a proper starting value for network point coordinates and $y_{1}^{[s]}, \ldots, y_{k}^{[s]}$ the result of the $s$ th step. The components of $y_{1}^{[s+1]}, \ldots, y_{k}^{[s+1]}$ are generated according the conditional distributions $D$,

$Z_{1} \sim D\left(\mathbf{Z} / y_{2}^{[s]}, \ldots, y_{k}^{[s]}\right) \quad Z_{2} \sim D\left(\mathbf{Z} / Z_{1}, y_{3}^{[s]}, \ldots, y_{k}^{[s]}\right)$

The iteration for the $k$ th component reads $Z_{k} \sim D\left(\mathbf{Z} / Z_{1}, \ldots Z_{k-1}\right)$. The vector $\left(Z_{1}, \ldots, Z_{k}\right)=\left(y_{1}^{[s+1]}, \ldots . y_{k}^{[s+1]}\right)$ serves as realization in the $s+1$ th step. The conditional distributions are one dimensional and therefore the algorithm does not change in structure if $k$ increases. While working with normal distributions, a data augmentation leads to conditionally independent variables. That ensures several convergence properties of the procedure as well.

In our network we introduced non-informative priors. These priors are based on the Fisher information and are not normalized. The conditional distributions can be obtained directly by the Gibbs sampler if we start the procedure within a certain region. Standard arguments of convergence of the Gibbs sampler fail in the case of non-informative priors. A monitoring of the simulation and the corresponding estimates is needed and we choose with this problem by using data sets as training samples. Therefore we reach a state of the procedure where a proper prior is given.

If the Bayesian estimate of the parameter is needed only instead of the complete posterior distribution a version of Gibbs sampling is carried out for computing the parameter estimates. Such algorithms are called EM-algorithm (Expectation Maximization) and are originally introduced to calculate maximum likelihood estimates. The stepwise calculation of a maximum likelihood estimate involves the cyclic 


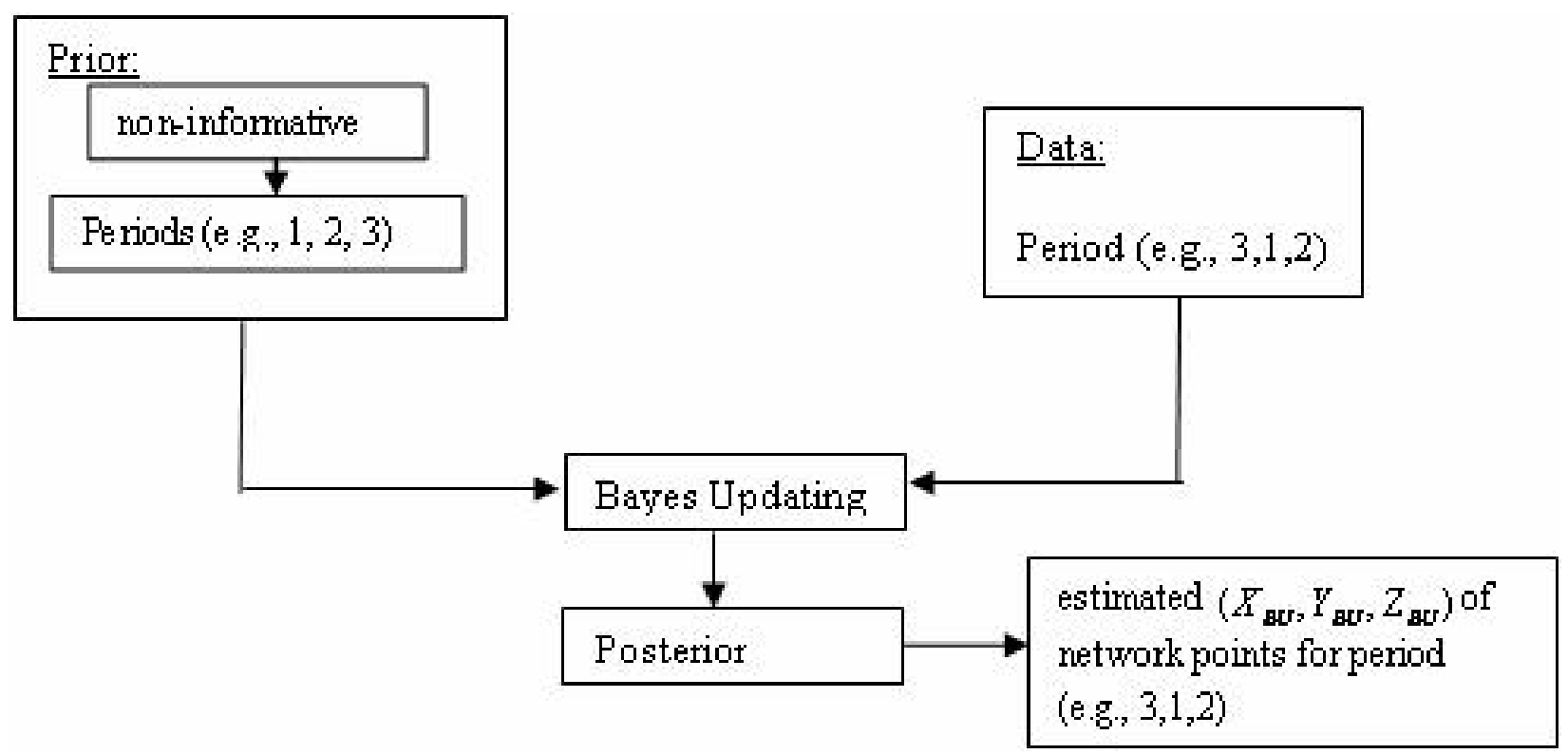

Fig. 3. Flowchart for BU method.

exchange of the parameters as in the above calculation combined with a maximization step (Felsenstein, 2001). If the prior distribution is non-informative maximum likelihood and Bayes estimation coincide for normally distributed data. The results of Bayesian estimates reduce to the calculation of the least square estimates if non-informative priors are used only.

\section{Application}

3.1 Information about the study area and individual adjustment results for different periods

Earthquakes and landslides are two most effective natural hazards in Turkey. The Eastern Black Sea region of Turkey receives a lot of rainfall and experienced very heavy flooding (Önalp, 1991; Tarhan, 1991; Ocakoglu et al., 2002). This region undergoes much more landslides compared to other regions of Turkey. Landslides on this region of Turkey damage agricultural areas, railways and cause the loss of life. Because of these reasons, scientists put this region as one of the most priority areas for their research. We selected Kutlugün Village in Macka County in the Province of Trabzon in Eastern Black Sea Region of Turkey as study area (Fig. 1). Kutlugün landslides damaged the Trabzon-Macka Highway and the Water Pipe Line supplying drinking water to Trabzon city-centre. Besides, lots of residential estates were affected during slow sliding. In order to prevent possible future damages of landslides at Kutlugün village, a geodetic deformation network was established in the year 2000 for landslide monitoring within the project of "Determination of landslides by dynamic models" by researchers from the Department of Geodesy and Photogrammetry Engineering at Karadeniz Technical University. In this study, repeated GPS surveys at certain time periods, belonging to a geodetic deformation network in Macka in the province of Trabzon in north eastern Turkey (Fig. 1) are used.

The network consists of 14 points, with four of them (2, 8,10 , and 13) on solid ground. The other points $(1,3,4$, $5,6,7,9,11,12$, and 14) were placed into moving material (Fig. 2). All of them were built with pillars. Geodetic deformation measurements were made in November 2000, February 2001 and May 2001 with Ashtech GPS receivers in a static mode. This data was evaluated by Geogenius- 2000 software. The outputs of this software are baselines $(l)$ between network points and cofactor matrices of each baseline $(Q)$ which were used also as input data in the studies of Yalcinkaya and Bayrak, 2003, 2005. With these outputs from the GPS software and approximate coordinates for network points, we can write our observation equations in the Gauss Markov Model (GMM) to estimate network point positions by least-squares estimation. The results from least-squares estimation are used as input data for Bayes-Updating and Gibbs-Sampling algorithms in this study.

The GMM is a linear mathematical model consisting of functional and stochastic relations. It relates observations to parameters. In matrix notation it takes the following form

$E(\boldsymbol{l})=\mathbf{A} \boldsymbol{x} \quad \boldsymbol{l}=\mathbf{A} \boldsymbol{x}+\varepsilon$

$E\left(\boldsymbol{\varepsilon} \varepsilon^{T}\right)=\Sigma=\sigma_{0}^{2} \mathbf{Q}$

where $\boldsymbol{l}$ is the $n \times 1$ vector of observations, $E($.$) is expecta-$ tion operator, A is the $n \times 3 u$ matrix of known coefficients, $\boldsymbol{\varepsilon}$ 
is the $n \times 1$ vector of errors, $\boldsymbol{x}$ is the $3 u \times 1$ vector of unknown parameters, $\Sigma$ is the $n \times n$ covariance-covariance matrix, $\mathbf{Q}$ is the $n \times n$ cofactor matrix of observations with $u$ number of network points and $n$ number of baselines. $\sigma_{0}^{2}$ is a priori variance factor. This theoretical model is rewritten to estimate parameters (network station coordinates) from real data (baselines between network points) in the following form

$l+v=\mathbf{A} \hat{\boldsymbol{x}} \quad \mathbf{P}=\mathbf{Q}^{-1}$

where $\mathbf{P}$ is the $n \times n$ weight matrix of observations; $\boldsymbol{v}$ is the $n \times 1$ vector of residuals. The estimation function $\hat{\boldsymbol{x}}=\left(\mathbf{A}^{T} \mathbf{P} A\right)^{-1}\left(\mathbf{A}^{T} \mathbf{P} l\right)$ is used to get the estimated values of network point coordinates (see Table 1 ).

$m_{p(i)}=\sqrt{m_{x(i)}^{2}+m_{y(i)}^{2}+m_{z(i)}^{2}}$ with $(i=1,2, \ldots, u)$ number point positioning error for network points are calculated by $\mathbf{Q}_{x x}=\left(\mathbf{A}^{T} \mathbf{P} A\right)^{-1}$ as $u \times u$ cofactor matrix of estimated parameters, $\mathbf{K}_{x x}=\sigma^{2} \mathbf{Q}_{x x} u \times u$ variance-covariance matrix of the estimated parameters and $m_{(x y z) i}=\sqrt{\left(\mathbf{K}_{x x}\right)_{i i}}$ with $(i=1,2, \ldots, u)$ mean-square errors for estimated coordinates. By looking at the $m_{p(i)}$ values of points, three different groups in each period are determined as accurate, more accurate and the most accurate. The starting points are selected from these groups. I.e., point 4 can be assigned to the first group with the smallest $m_{p}$ value, point 3 to the second group, and point 13 to the third group with nearly the biggest $m_{p}$ value at every period. The grey rows in Table 2 are used to express the points which are selected as starting points according to their $m_{p(i)}$ values. The starting points are selected as 1, 3, 4, and 13 (see Table 2). Different point sequences which are constructed according to such a grouping for network points are used for Gibbs Sampling algorithm later.

3.2 Comparison between different bayes updating and gibbs sampling algorithms

The parameter estimation with Gibbs-Sampling is applied with different starting points and with Bayes updating with different prior information (Roberts and Rosenthal, 1998). The results obtained with different information (starting points and prior information) in two different algorithms are compared. Coordinate parameters estimated from GibbsSampling (GS) algorithm and Bayes-Updating (BU) are called as $\left(\boldsymbol{X}_{\mathrm{GS}}, \boldsymbol{Y}_{\mathrm{GS}}, \boldsymbol{Z}_{\mathrm{GS}}\right)$ and $\left(\boldsymbol{X}_{\mathrm{BU}}, \boldsymbol{Y}_{\mathrm{BU}}, \boldsymbol{Z}_{\mathrm{BU}}\right)$. Figures 3 and 4 show flowcharts of BU and GS methods respectively.

Parameters of the differences are calculated; one is the difference between Bayes-Updating and Gibbs-Sampling algorithms (BU-GS), one is between Gibbs-Sampling algorithms and the other is Bayes-Updating algorithms. These differences are calculated as

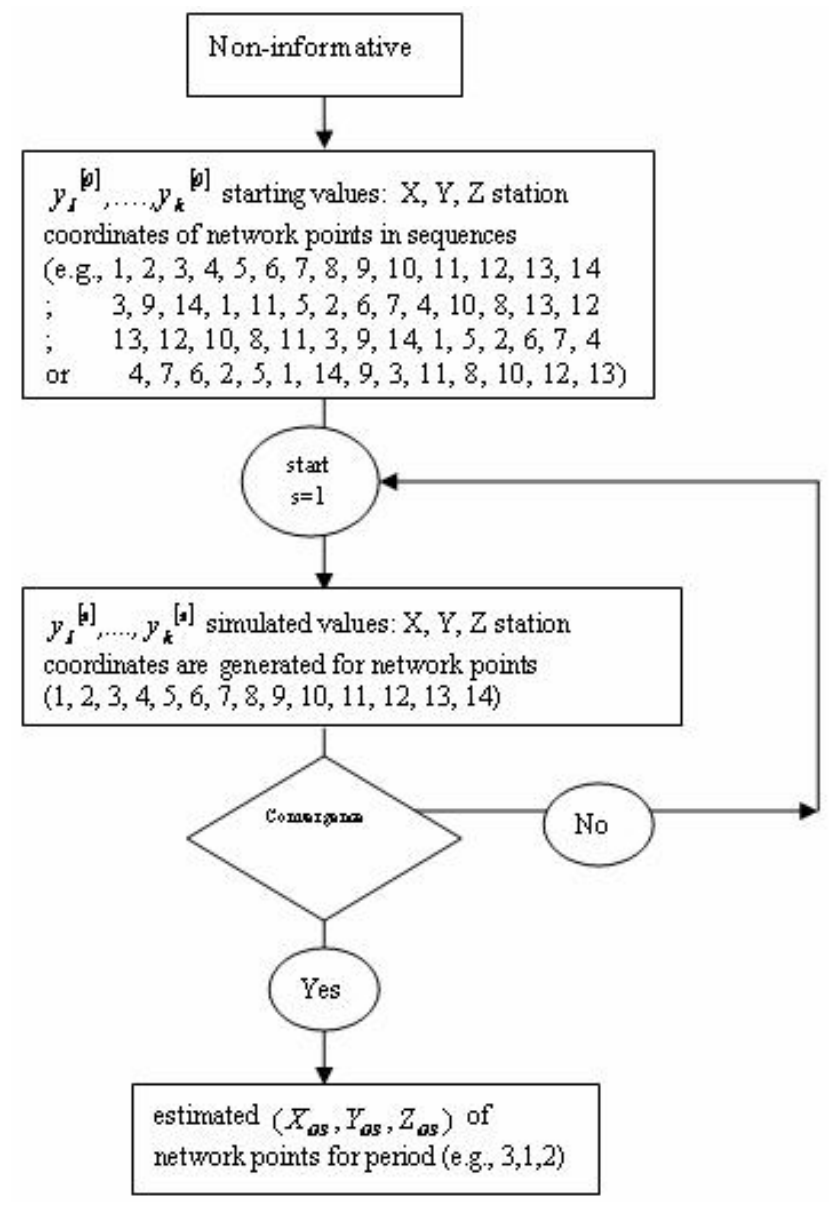

Fig. 4. Flowchart for GS method.

Here, $i$ and $k$ indicate the starting points in GibbsSampling algorithm (e.g., 1, 3, 4, or 13); $j$ and $l$ indicate prior information (e.g., the first, second or third period) and $\mathrm{n}$ indicates the number of points in the network. The results are drawn (in Figs. 5 and 6) except for GS. Comparisons are made by using different types of prior information in Bayes updating and taking different starting points $(4,3,13)$ in Gibbs-Sampling algorithm. For a comparison between the different algorithms, the smoothness parameters $(V R)$ are calculated on values of different algorithms. $f(1), f(2), \ldots \ldots, f(n)$ values are taken as $\left(\boldsymbol{B} U_{-} G S\right)$ and $(\boldsymbol{B} U)$ values and (VR) values for all plots are calculated from $\mathrm{VR}=\sum_{i=2}^{n}|f(i)-f(i-1)|$.

For the first period, the differences on all points are approximately the same except of point 1 (see Table 3 ). Because the VR values are nearly the same as for all situations, 


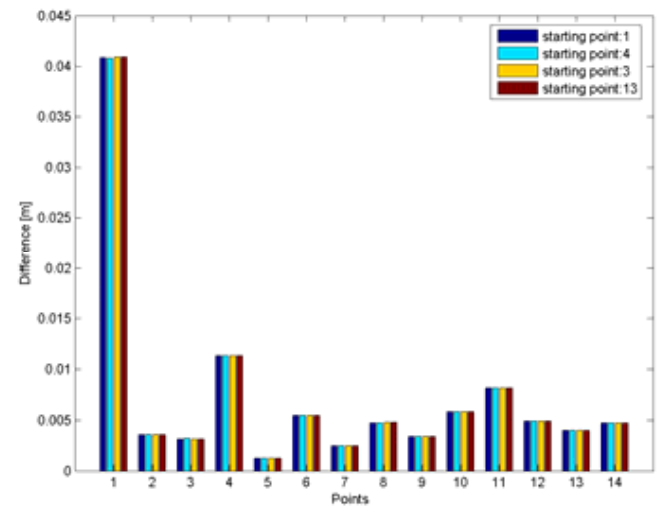

(a) BU with the second period as prior and GS with different starting points in the first period

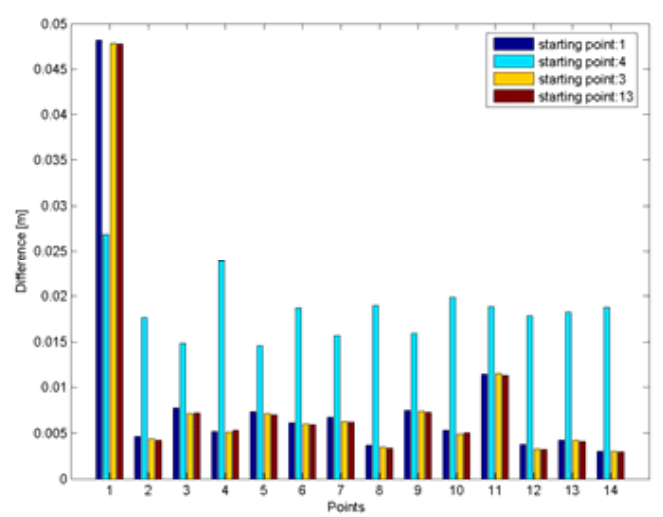

(c) BU with the first period as prior and GS with different starting points in the second period

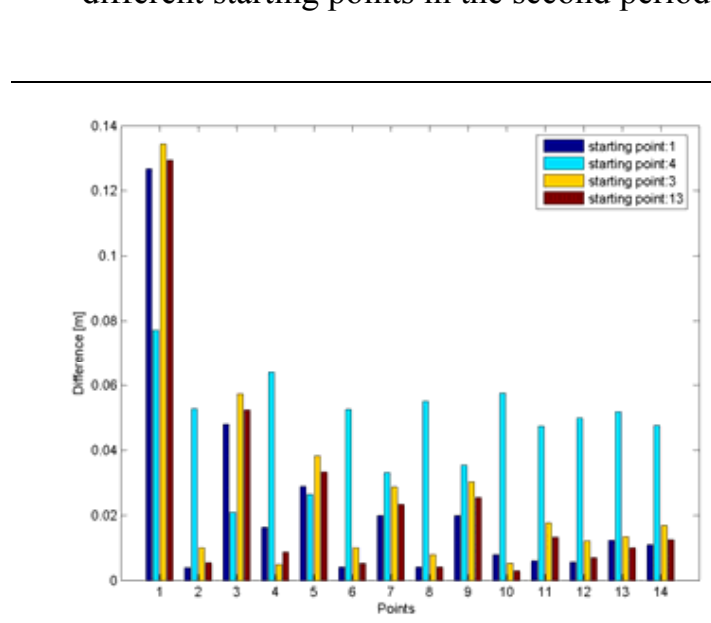

(e) BU with the first period as prior and GS with different starting points in the third period

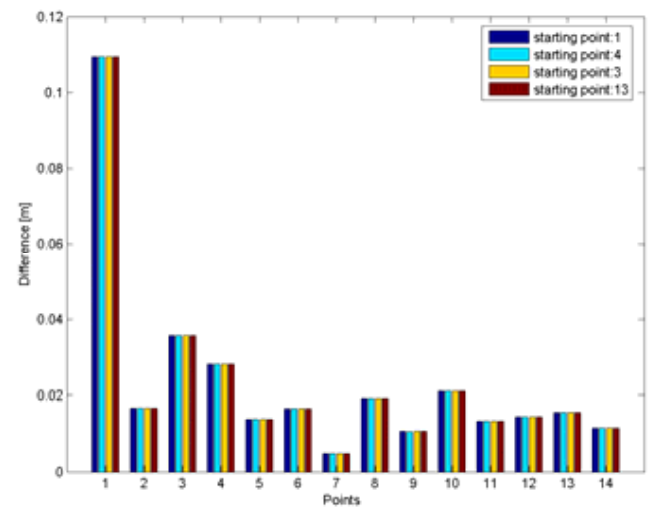

(b) BU with the third period as prior and GS with different starting points in the first period

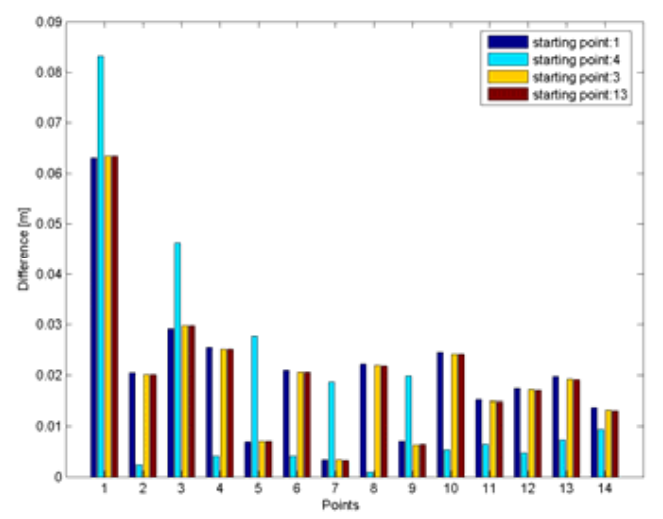

(d) BU with the third period as prior and GS with different starting points in the second period

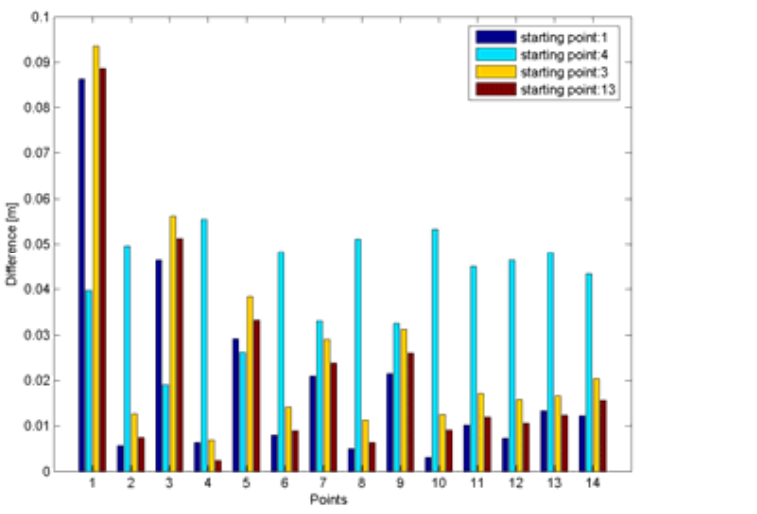

(f) BU with the second period as prior and GS with different starting points in the third period

Fig. 5. Differences between BU and GS Results. 
Table 1. Adjusted station coordinates for each period.

\begin{tabular}{|c|c|c|c|c|}
\hline No & $X(\mathrm{~m})$ & $Y(\mathrm{~m})$ & $Z(\mathrm{~m})$ & \\
\hline 1 & 3710656.9536 & 3083935.0997 & 4157818.3179 & \multirow{21}{*}{ November 2000 (1. period) } \\
\hline 2 & 3710501.7100 & 3084005.1000 & 4157907.9599 & \\
\hline 3 & 3710542.0823 & 3084033.1843 & 4157788.0178 & \\
\hline 4 & 3710709.5387 & 3084028.6271 & 4157648.6440 & \\
\hline 5 & 3710480.9725 & 3084117.3939 & 4157741.7704 & \\
\hline 6 & 3710274.8591 & 3084218.3811 & 4157799.2949 & \\
\hline 7 & 3710479.6401 & 3084171.0299 & 4157677.5811 & \\
\hline 8 & 3710205.6285 & 3084212.3196 & 4157845.5019 & \\
\hline 9 & 3710389.3907 & 3084138.4578 & 4157788.8566 & \\
\hline 10 & 3710292.4179 & 3084153.5430 & 4157861.5580 & \\
\hline 11 & 3710442.6596 & 3084257.8606 & 4157623.1696 & \\
\hline 12 & 3710342.5861 & 3084401.6708 & 4157575.3478 & \\
\hline 13 & 3710193.7381 & 3084419.1729 & 4157702.3677 & \\
\hline 14 & 3710280.1589 & 3084340.5894 & 4157711.3664 & \\
\hline 1 & 3710656.8976 & 3083935.1534 & 4157818.2912 & \\
\hline 2 & 3710501.7145 & 3084005.0948 & 4157907.9607 & \\
\hline 3 & 3710542.0876 & 3084033.1875 & 4157788.0150 & \\
\hline 4 & 3710709.558 & 3084028.6265 & 4157648.6582 & \\
\hline 5 & 3710480.9714 & 3084117.3930 & 4157741.7718 & \\
\hline 6 & 3710274.8622 & 3084218.3707 & 4157799.2936 & \\
\hline 7 & 3710479.6437 & 3084171.0287 & 4157677.5780 & \\
\hline 8 & 3710205.6330 & 3084212.3114 & 4157845.5022 & \multirow{21}{*}{ February 2001 (2. period) } \\
\hline 9 & 3710389.3879 & 3084138.4516 & 4157788.8570 & \\
\hline 10 & 3710292.4275 & 3084153.5456 & 4157861.5672 & \\
\hline 11 & 3710442.6560 & 3084257.8451 & 4157623.1631 & \\
\hline 12 & 3710342.5935 & 3084401.6693 & 4157575.3520 & \\
\hline 13 & 3710193.7406 & 3084419.1657 & 4157702.3702 & \\
\hline 14 & 3710280.1649 & 3084340.5867 & 4157711.3736 & \\
\hline 1 & 3710656.8154 & 3083935.2621 & 4157818.2686 & \\
\hline 2 & 3710501.7336 & 3084005.0773 & 4157907.9688 & \\
\hline 3 & 3710542.0759 & 3084033.2138 & 4157788.9525 & \\
\hline 4 & 3710709.5795 & 3084028.6021 & 4157648.6741 & \\
\hline 5 & 3710480.9483 & 3084117.4034 & 4157741.7636 & \\
\hline 6 & 3710274.8795 & 3084218.3582 & 4157799.3071 & \\
\hline 7 & 3710479.6411 & 3084171.0348 & 4157677.5734 & \\
\hline 8 & 3710205.6520 & 3084212.2917 & 4157845.5143 & \\
\hline 9 & 3710389.3739 & 3084138.4486 & 4157788.8531 & \\
\hline 10 & 3710292.4434 & 3084153.5157 & 4157861.5777 & \\
\hline 11 & 3710442.6683 & 3084257.8356 & 4157623.1695 & \\
\hline 12 & 3710342.6079 & 3084401.6544 & 4157575.3566 & \\
\hline 13 & 3710193.7515 & 3084419.1573 & 4157702.3914 & \\
\hline 14 & 3710280.1660 & 3084340.5752 & 4157711.3833 & \\
\hline
\end{tabular}

it can be concluded for the first period that there is no difference of using different starting points in BU algorithm (see Figs. 5a, 3b).

In case using the first period as a prior in BU for the second period, the differences on all points are approximately the same except for point 1 . Figure $5 \mathrm{c}$ shows that the differences between GS with starting point 4 and BU become higher for all points except point 1 . On the other hand, there is nearly no difference between $\mathrm{BU}$ and GS with the starting points respectively 1,3 and 13 (see Fig. 5c). In case of using the third period as a prior in updating for the same period, the situation becomes different from the previous case. That is, point 1 gives the biggest value for the difference between GS with the starting point 4 and BU (see Fig. 5d).

Figure $5 \mathrm{e}$ denotes that using of the first period as a prior in BU for third period discloses more or less the same behaviours of the second period (see Fig. 5c) concerning the differences which depend on starting points. 
Table 2. $\left(m_{p} \mathrm{~cm}\right)$ point positioning errors for the all network points calculated from individual adjustment results in each period.

\begin{tabular}{llll}
\hline Points & 1. period $\left(\mathrm{m}_{p} \mathrm{~cm}\right)$ & 2. period $\left(\mathrm{m}_{p} \mathrm{~cm}\right)$ & 3. period $\left(\mathrm{m}_{p} \mathrm{~cm}\right)$ \\
\hline 1 & 0.178 & 0.205 & 0.237 \\
2 & 0.171 & 0.225 & 0.266 \\
3 & 0.183 & 0.247 & 0.269 \\
4 & 0.150 & 0.175 & 0.207 \\
5 & 0.172 & 0.257 & 0.269 \\
6 & 0.160 & 0.243 & 0.263 \\
7 & 0.154 & 0.208 & 0.246 \\
8 & 0.265 & 0.340 & 0.383 \\
9 & 0.182 & 0.249 & 0.309 \\
10 & 0.217 & 0.344 & 0.286 \\
11 & 0.196 & 0.201 & 0.251 \\
12 & 0.273 & 0.283 & 0.324 \\
13 & 0.312 & 0.277 & 0.371 \\
14 & 0.179 & 0.231 & 0.273 \\
\hline
\end{tabular}

Table 3. VR values for differences between BU and GS Results (cm).

\begin{tabular}{|c|c|c|c|c|c|c|c|}
\hline prior period & start point & prior period & start point & prior perioc & start point & prior period & start point \\
\hline \multicolumn{8}{|c|}{ VR values: BU-GS differences $(\mathrm{m})$ for 1 . period } \\
\hline 2. period & 1 & 2. period & 4 & 2. period & 3 & 2. period & 13 \\
\hline \multicolumn{2}{|c|}{0.07653} & \multicolumn{2}{|c|}{0.07636} & \multicolumn{2}{|c|}{0.07659} & \multicolumn{2}{|c|}{0.07659} \\
\hline 3. period & 1 & 3. period & 4 & 3. period & 3 & 3. period & 13 \\
\hline \multicolumn{2}{|c|}{0.19574} & \multicolumn{2}{|c|}{0.19577} & \multicolumn{2}{|c|}{0.19576} & \multicolumn{2}{|c|}{0.19576} \\
\hline \multicolumn{8}{|c|}{ VR values: BU-GS differences $(\mathrm{m})$ for 2. period } \\
\hline 1. period & 1 & 1. period & 4 & 1. period & 3 & 1. period & 13 \\
\hline \multicolumn{2}{|c|}{0.0780} & \multicolumn{2}{|c|}{0.0511} & \multicolumn{2}{|c|}{0.0780} & \multicolumn{2}{|c|}{0.0770} \\
\hline 3. period & 1 & 3. period & 4 & 3. period & 3 & 3. period & 13 \\
\hline \multicolumn{2}{|c|}{0.1761} & \multicolumn{2}{|c|}{0.2870} & \multicolumn{2}{|c|}{0.1777} & \multicolumn{2}{|c|}{0.1775} \\
\hline \multicolumn{8}{|c|}{ VR values: BU-GS differences $(\mathrm{m})$ for 3. period } \\
\hline 1. period & 1 & 1. period & 4 & 1. period & 3 & 1. period & 13 \\
\hline \multicolumn{2}{|c|}{0.3064} & \multicolumn{2}{|c|}{0.2646} & \multicolumn{2}{|c|}{0.3956} & \multicolumn{2}{|c|}{0.3713} \\
\hline 2. period & 1 & 2. period & 4 & 2. period & 3 & 2. period & 13 \\
\hline \multicolumn{2}{|c|}{0.2862} & \multicolumn{2}{|c|}{0.2160} & \multicolumn{2}{|c|}{0.3113} & \multicolumn{2}{|c|}{0.3066} \\
\hline
\end{tabular}

In case of using second period as a prior in BU for the third period, the biggest difference can be found also on point 1 with the value nearly $9 \mathrm{~cm}$. With the starting point 4 , the differences on all points become higher except point 1 (see Fig. 5f).

\subsection{Comparison between different gibbs sampling algo-} rithms

As indicated in Sect. 3.1., all network points are divided into groups according to their point positioning errors $\left(m_{p}\right)$ values and this grouping is used for GS Algorithm, to decide on which point to be started. For the first period, there is no difference using different starting points. For the second period, there is a big difference between the normal case (take the points in ascending order respectively $1,2,3, . ., 14$ ) and the case 4 (using point 4 as starting). On the other hand, there is slight difference among the normal case and 3 and 13 cases. The same inferences can be made for the third period. However, the differences are bigger than the second period. E.g. the difference of the normal case and the case 4 is nearly $2 \mathrm{~cm}$ in the second period. This difference is nearly $5 \mathrm{~cm}$ in the third period. As a result, it can be said that from the most to least effective starting points are respectively 4 , 
3, 13. However, the degree of effectiveness of point 3 and point 13 are nearly the same.

3.4 Comparison between different bayes updating algorithms

In Bayes Updating algorithm, it is an important question which prior information is most informative compared to others. To answer this question in this study, inference should be made in different periods with different prior information. For every algorithm, VR are also calculated. Differences between these algorithms in the aspects of different prior information are following:

By using of non-informative information and the second period as a prior in the first period, the biggest difference is determined on point 1 with the value $4 \mathrm{~cm}$. The smallest difference on point 5 with the value $0.03 \mathrm{~cm}$ is determined. For the same period, by using of non-informative information and the third period as a prior, the biggest difference is determined on point 1 with the value $13 \mathrm{~cm}$. The smallest difference on point 7 is $0.4 \mathrm{~cm}$. Finally, the biggest difference is determined on point 1 with using the second period and the third period as priors, see Fig. 6 a.

For the second period by using non-informative information and the first period as a prior we get the biggest difference on point 1 with the value $4.72 \mathrm{~cm}$ and the smallest difference on point 5 with the value $0.14 \mathrm{~cm}$. By using noninformative information and the third period as a prior, the biggest difference is determined on point 1 with the value $8.73 \mathrm{~cm}$ and the smallest difference on point 7 with the value $0.42 \mathrm{~cm}$. For the same period, the first period and the third period are used as prior, the biggest difference is determined on point 1 with the value $13.42 \mathrm{~cm}$ and the smallest difference on point 7 with the value $0.24 \mathrm{~cm}$ (see Fig. 6b). Almost the same procedures are done for the third period to evaluate Bayes Updating algorithm with different prior information. When we use non-informative information and the first period as a prior, we calculate the biggest difference on point 1 with the value $13.41 \mathrm{~cm}$ and the smallest difference on point 7 with the value $0.33 \mathrm{~cm}$. In case of using non-informative information and the second period as a prior, the biggest difference is determined on point 1 with the value $8.71 \mathrm{~cm}$. The smallest difference can be found on point 7 with the value $0.50 \mathrm{~cm}$. For the same period, first period and the second period were used as priors. This prior information let the biggest difference be on point 1 with the value $4.74 \mathrm{~cm}$ and the smallest difference on point 5 with the value $0.08 \mathrm{~cm}$ (see Table 4 and Fig. 6c).

3.5 Test of hypotheses for differences between different settings of methods and deformation between the measurement periods

In this section we compare the results obtained by different statistical methods described in previous sections. The

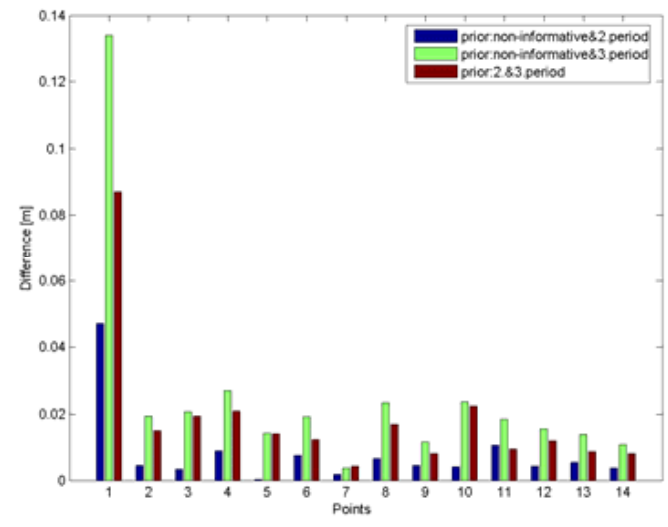

(a) BU by different prior information in the first period

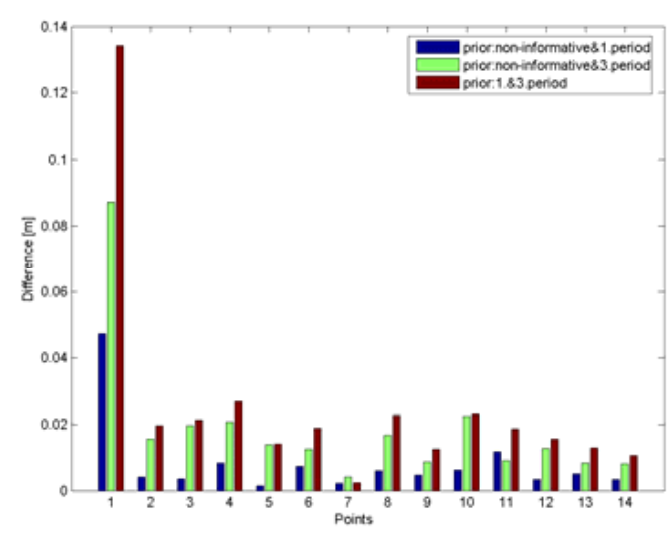

(b) BU by different prior information in the second period

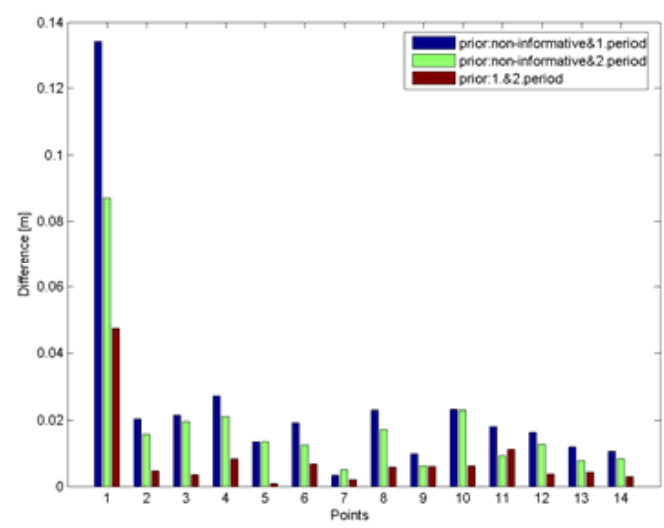

(c) BU by different prior information in the third period

Fig. 6. Differences between BU algorithms.

main question we discuss is whether the method chosen major influence on the outcome or not. The different methods are examined pairwise on equal estimations of parameters. Therefore we apply a multivariate test for equal mean vectors of parameter estimations. Again, we examine this hypothesis testing problem from a Bayesian viewpoint. The prior 
Table 4. VR values for differences between different setting of BU algorithms $(\mathrm{cm})$.

\begin{tabular}{|c|c|c|c|c|}
\hline \multicolumn{5}{|c|}{ BU differences for 1. period } \\
\hline \multicolumn{2}{|c|}{ Prior period } & \multicolumn{2}{|c|}{ Prior period } & Prior period \\
\hline $\begin{array}{r}\text { non- informative } \\
0.0935\end{array}$ & 2. period & $\begin{array}{r}\text { non- informative } \\
0.2103\end{array}$ & 3. period & $\begin{array}{l}\text { 1. period 3. period } \\
0.1495\end{array}$ \\
\hline \multicolumn{5}{|c|}{ BU differences for 2. period } \\
\hline $\begin{array}{r}\text { non- informative } \\
0.0902\end{array}$ & 1. period & $\begin{array}{r}\text { non- informative } \\
0.1494\end{array}$ & 3. period & $\begin{array}{l}\text { 1. period 3. period } \\
0.2094\end{array}$ \\
\hline \multicolumn{5}{|c|}{ BU differences for 3. period } \\
\hline $\begin{array}{r}\text { non- informative } \\
0.2138\end{array}$ & 1. period & $\begin{array}{r}\text { non-informative } \\
0.1551\end{array}$ & 2. period & $\begin{array}{c}\text { 1. period 2. period } \\
0.0855\end{array}$ \\
\hline
\end{tabular}

Table 5. $p$-values for differences between different settings of BU algorithms.

\begin{tabular}{lllll}
\hline Period & \multicolumn{2}{c}{ Prior information } & $p$-value & decision about difference \\
\hline \multirow{3}{*}{ 1. period } & 2. period & non-informative & 1.0 & NO \\
& 3. period & non-informative & 2e-05 & YES \\
& 3. period & 2. period & 0.27224 & NO \\
\multirow{5}{*}{ 2. period } & 1. period & non-informative & 0.73809 & NO \\
& 3. period & non-informative & 0.0 & YES \\
& 3. period & 1. period & 0.0 & YES \\
\multirow{3}{*}{ 3. period } & 1. period & non-informative & 0.00023 & YES \\
& 2. period & non-informative & 0.47811 & NO \\
& 2. period & 1. period & 1.0 & NO \\
\hline
\end{tabular}

distribution used is a Normal-Whishart distributions introduced as conjugate prior for the network structure in Sect. 1. The prior information is established in the same way as in the case of parameter estimation. Starting with vague prior the data of period 1, period 2 or period 3 are used as learning samples in turn.

Vague priors might be included in the range of conjugate priors in the sense that the vague prior is found as a limit of proper conjugate priors. Using vague priors for the parameters, we find that the Bayesian testing statistics approaches classical testing statistics. The basis of the Bayesian decision $B_{F}$ gives the Bayes-factor as follows

$B_{F}=\frac{m\left(D \mid H_{0}\right)}{m\left(D \mid H_{1}\right)}$

From a Bayesian context, the Bayes factor represents the testing statistics, that is the ratio of the marginal likelihoods on the assumption of the null-hypothesis and alternative hypothesis respectively. The marginals follow Eq. (5), note that in case of the alternative hypothesis the dimension of the multivariate Normal distribution differs from the case of null-hypothesis. Methodological details of Bayesian testing in a multivariate model are explained in Rowe (2002).

Actually, the Bayes factor shows the results of tests. Anyway, Tables 5, 6 and 7 show $p$-values representing the result as well as for classical tests. Classical p-value, defined from a point null hypothesis, can be generalized in various ways. The p-value will be understood as a posterior $\mathrm{p}$-value. That is the probability, given the data, that a future observation is more extreme (as measured by some test variable) than the data. Since we assessed vague priors in most cases our pvalue makes little difference to the classical p-value.

In order to decide whether we have significant deformation or not between the measurement periods (e.g., between period 1 - period 2 or between period 2- period 3 ), the $p$-values are calculated on the assumption of the nullhypothesis which accept there is no deformation between measurement period and alternative hypothesis which contradict with the null-hypothesis. The corresponding $p$-values for deformation analysis and decision about the deformation are given in Tables 8 and 9 . 
Table 6. $p$-values for difference between different settings of GS algorithms.

\begin{tabular}{lllll}
\hline Period & starting point & $p$-value & decision about difference \\
\hline & 1 & 3 & 1.00000 & NO \\
& 1 & 4 & 1.00000 & NO \\
1. period & 1 & 13 & 1.00000 & NO \\
& 3 & 13 & 1.00000 & NO \\
& 4 & 3 & 1.00000 & NO \\
& 4 & 13 & 1.00000 & NO \\
& 1 & 3 & 1.00000 & NO \\
& 1 & 4 & 0.00002 & YES \\
& 1 & 13 & 1.00000 & NO \\
& 3 & 13 & 1.00000 & NO \\
& 4 & 3 & 0.00015 & YES \\
& 4 & 13 & 0.00015 & YES \\
& 1 & 3 & 1.00000 & NO \\
& 1 & 4 & 0.00000 & YES \\
& 1 & 13 & 1.00000 & NO \\
& 3 & 13 & 1.00000 & NO \\
& 4 & 3 & 0.00000 & YES \\
& 4 & 13 & 0.00000 & YES \\
\hline
\end{tabular}

Table 7. $p$-values for difference between different settings of BU and GS algorithms.

\begin{tabular}{|c|c|c|c|c|}
\hline Period & prior information & starting point & $p$-value & decision about difference \\
\hline \multirow{9}{*}{ 1.period } & 2. period & 1 & 0.99999 & NO \\
\hline & 2. period & 3 & 0.99999 & NO \\
\hline & 2. period & 4 & 0.99999 & NO \\
\hline & 2. period & 13 & 0.99999 & NO \\
\hline & 3.period & 1 & 0.00000 & YES \\
\hline & 3. period & 3 & 0.00000 & YES \\
\hline & 3. period & 4 & 0.00000 & YES \\
\hline & 3. period & 13 & 0.00000 & YES \\
\hline & 1. period & 1 & 0.28182 & $\mathrm{NO}$ \\
\hline \multirow{7}{*}{ 2.period } & 1. period & 3 & 0.36919 & NO \\
\hline & 1. period & 4 & 0.00160 & YES \\
\hline & 1. period & 13 & 0.37704 & NO \\
\hline & 3. period & 1 & 0.00000 & YES \\
\hline & 3. period & 3 & 0.00000 & YES \\
\hline & 3. period & 4 & 0.00000 & YES \\
\hline & 3. period & 13 & 0.00000 & YES \\
\hline \multirow{8}{*}{ 3.period } & 1. period & 1 & 0.00000 & YES \\
\hline & 1. period & 3 & 0.00000 & YES \\
\hline & 1. period & 4 & 0.00000 & YES \\
\hline & 1. period & 13 & 0.00000 & YES \\
\hline & 2. period & 1 & 0.01427 & YES \\
\hline & 2. period & 3 & 0.00048 & YES \\
\hline & 2. period & 4 & 0.00000 & YES \\
\hline & 2. period & 13 & 0.01295 & YES \\
\hline
\end{tabular}


Table 8. $p$-values calculated with the results from Bayes-Uptading for deformation analysis.

\begin{tabular}{lllll}
\hline Method & $\begin{array}{l}\text { measurement periods subject } \\
\text { of the deformation }\end{array}$ & $\begin{array}{l}\text { prior information used } \\
\text { in BU for each period }\end{array}$ & $p$-values & decision for deformation \\
\hline & 1. period-2. period & non-informative & 0.00072 & YES \\
Bayes-Updating (BU) & 1. period-2. period & 3. period & 0.99785 & NO \\
& 2. period-3. period & non-informative & 0.00000 & YES \\
& 2. period-3. period & 1. period & 0.00016 & YES \\
\hline
\end{tabular}

Table 9. $p$-values calculated with the results from Gibbs-Sampling for deformation analysis.

\begin{tabular}{lllll}
\hline Method & $\begin{array}{l}\text { measurement periods subject } \\
\text { of the deformation }\end{array}$ & $\begin{array}{l}\text { starting points used in } \\
\text { GS for each period }\end{array}$ & $p$-values & decision for deformation \\
\hline & 1. period-2. period & 13 & 0.04420 & YES \\
1. period-2. period & 1 & 0.03645 & YES \\
& 1. period-2. period & 3 & 0.04328 & YES \\
1. period-2. period & 4 & 0.00603 & YES \\
Gibbs-Sampling (GS) & 2. period-3. period & 13 & 0.00000 & YES \\
& 2. period-3. period & 1 & 0.00000 & YES \\
2. period-3. period & 3 & 0.00000 & YES \\
2. period-3. period & 4 & 0.00000 & YES \\
\hline
\end{tabular}

\section{Conclusions}

In this paper we introduced two estimation procedures "Bayes-Updating" and "Gibbs-Sampling" based on Bayes theory for deformation monitoring networks which allows accounting for prior information about the coordinate parameters. Gibbs-Sampling applies the simulation for the conditional distributions of parameter estimation of deformation network points. In Bayes-Updating algorithm, we can analyze the sensitivity of the model concerning the prior information coming from different epoch measurements in deformation network. In order to justify that the prior information is worth to consider, the significance test is applied on the Bayes-Updating results which varies according to different prior information. Concerning usage of these two algorithms, Gibbs-Sampling algorithm takes more time compared to Bayes-Updating because simulation algorithm of Gibbs-Sampling depends on the information about the quality of starting points itself. In this study we use a network with 14 points, when the user increase the number of network points, Gibbs-Sampling becomes complicated for such networks.

Comparing the results of the two parameter estimation procedures we get differences of network point coordinates on $\mathrm{cm}$ level. When we consider all these parameter estimation results as input data for deformation analysis, it should be pointed out here that the estimation procedure which is used in individual epochs has also big importance as deformation analysis procedures itself.
The results of significance test for difference between different setting of GS algorithm show that the estimate is sensitive to the choice of starting point, for example starting point 4 leads to difference in the results, see Table 6 . The differences for BU algorithms indicate that third period as a prior information leads to differences on the results more than the other periods, see Table 5 for significance test and Table 4 for variances. From the comparison between BU and GS algorithms, we conclude that third period as a prior information in BU algorithm and starting point 4 in GS algorithm leads significance of difference on the results. It should be mentioned here third period as a data in BU leads to significance of difference w.r.t. any prior information, see Table 7 for significance test and Table 3 for variance. The hypothesis test for deformation out of BU results exhibits significance of difference between first period-second period and second period-third period except in case of using third period as a prior information, see Table 8 . Therefore, we can conclude from this study that the prior information has impact on the results of BU algorithm. The same hypothesis test out of GS results indicates also deformation with evident deformation effect between second period-third period and less significant testing results by $p$-values near to the critical value 0.05 , see Table 9. The season which our 2. period (February) and 3.period (May) measurements corresponded to is a danger season with spring rainfall and snow melting in East Black Sea Region for landslides. The significance deformation which we got out of our testing for 2. period and 3.period might be a result of this. 
Acknowledgements. This study was done during the first author's research stay at the Institute of Statistics and Probability Theory at Vienna University of Technology (TU Vienna) and financially supported by Natural Science Institute of Karadeniz Technical University and TU Vienna. The first author is thankful to R. Viertl, head of the Statistics and Probability Theory Institute in TU Vienna, for providing her full scientific facilities during her research stay at this institute.

Edited by: Kang-tsung Chang

Reviewed by: three anonymous referees

\section{References}

Albertella, A., Cazzaniga, N., Sansò, F., Sacerdote, F., Crespi, M., and Luzietti, L.: Deformations detection by a Bayesian approach: prior information representation and testing criteria definition, ISGDM2005 - IAG Symposium volume n. 131, 2005.

Caspary, W. F.: Concept of network and deformation analysis, Monograph 11, School of Geomatics Engineering, The University of New South Wales, Australia, 2000.

Felsenstein, K.: Mathematische methoden für die interpretation von risiken, Imago Hominis, 11, 261-269, 2004.

Felsenstein, K.: Bayesian interpolation schemes for monitoring systems. In: Advances in model-oriented design and analysis, 6, Physica-Verlag, Heidelberg, 2001.

Felsenstein, K.: Bayes'sche statistik für kontrollierte experimente, Vandenhoeck and Ruprecht, Göttingen, 1996.
Koch, K. R.: Bayesian Inference with Geodetic Applications, Springer, Berlin, Heidelberg, New York, 1990.

Ocakoglu, F., Gkceoglu, C., and Ercanoglu, M.: Dynamics of a Complex Mass Movements Triggered by Heavy Rainfall: A Case Study from NW Turkey, Geomorphology, 42, 329-341, 2002.

Önalp, A.: Landslides of East Black Sea Region - Reasons, Analysis and Controls, 1st National Landslides Symposium of Turkey, Trabzon, Proceedings Paper, 85-95, (in Turkish), 1991.

Robert, C. and Casella, G.: Monte Carlo statistical methods Springer, New York, 2004.

Robert, C.: The Bayesian Choice, Springer, New York, 2001.

Roberts, G. and Rosenthal, J.: Markov chain Monte Carlo: Some practical implications of theoretical results, Canadian J. Statist., 25, 5-32, 1998.

Rowe, D.B.: Multivariate Bayesian Statistics, Chapman and Hall/CRC, London, 2002.

Tarhan, F.: A look to landslides of East Black Sea Region, 1st National Landslides Symposium of Turkey, Trabzon, Proceedings Paper, 38-63 (in Turkish), 1991.

Yalcinkaya, M. and Bayrak, T.: Dynamic model for monitoring landslides with emphasis on underground water in Trabzon province, Northeastern Turkey, Journal of Surveying Engineering, August 2003, 129(3), 115-124. 2003.

Yalcinkaya M., Tanir, E.: A study on using Bayesian statistics in geodetic deformation analysis, Proceedings 11th International FIG Symposium on Deformation Measurements, Santorini (Thera) Island, Greece, 25-28 May 2003, 2003.

Yalcinkaya, M. and Bayrak, T.: Comparison of static, kinematic and dynamic geodetic deformation models for Kutlugün landslide in northeastern Turkey, Natural Hazards, January 2005, 34(1), 91110, 2005. 